\title{
Flow and geometrical effects on radiated noise from exhausts computed by a hybrid extended Fourier PSTD method
}

\author{
M. Hornikx, ${ }^{*}$ W. De Roeck, ${ }^{*}$ and W. Desmet ${ }^{\dagger}$ \\ K.U. Leuven, Dept. of Mechanical Engineering, Celestijnenlaan 300, B-3001 Leuven, Belgium
}

\begin{abstract}
In urban areas, a significant source of noise from road traffic is the contribution by the sound radiated from exhaust pipes. The current work has initialized a systematic numerical study on the combined effects of the geometry surrounding the exhaust pipe, i.e. the ground surface and the automotive body, as well as the influence of the non-uniform velocity and temperature from the exhaust jet on the directivity and power radiated from the pipe. The problem is treated by first solving the RANS equations to obtain the mean velocity and temperature solution of the exhaust jet. Then, the acoustic radiation is computed by solving the linearized Euler (LEE) equations. For an efficient solution of the LEE, a recently developed multi-domain extended Fourier pseudospectral time-domain (PSTD) methodology is used. This method combines the favorable spectral accuracy of the PSTD method with a local grid refinement in the region with high gradients of the mean flow and temperature fields. The numerical methodology is successfully verified for a case with a hot flow by a comparison with a reference numerical methodology. The presence of a rigid ground surface and simplified automotive body is shown to increase the radiated sound power by $6 \mathrm{~dB}$ for the lower frequency region, and the effect of the body on the directivity is largest for the higher frequencies. Flow effects slightly increase the shielding effect of the body for all frequencies, but have a main impact behind the exhaust pipe, where low frequencies experience higher levels and a cone of low sound levels characterizes the high frequencies.
\end{abstract}

\section{Nomenclature}

Symbols

$\delta_{i j} \quad$ Kronecker delta

$\delta \quad$ Dirac delta function

$\Delta x \quad$ Spatial discretization, $\mathrm{m}$

$\gamma \quad$ Heat capacity ratio

$\theta \quad$ Angle in vertical plane, deg.

$\rho \quad$ Density, $\mathrm{kg} / \mathrm{m}^{3}$

$\phi \quad$ Angle in horizontal plane, deg.

$\Omega \quad$ Computational domain

Subscripts

0 Ambient variable

c Coarse grid

f Fine grid

i Summation variable

in Incident

j 1/3-octave band index

p Pressure

rad Radiated
Half exhaust pipe width, $m$

Constant in LEE source function, $\mathrm{s}^{2}$

Adiabatic sound speed, $\mathrm{m} / \mathrm{s}$

Frequency, $\mathrm{Hz}$

Green's function

Wavenumber, $\mathrm{m}^{-1}$

Mach number

Pressure, $\mathrm{N} / \mathrm{m}^{2}$

Acoustic variable vector

Distance to receiver, $\mathrm{m}$

Signum function

Time, s

Temperature, K

Power, Watt

Velocity vector, $\mathrm{m} / \mathrm{s}$

*Post-doc, maarten.hornikx@mech.kuleuven.be

${ }^{\dagger}$ Professor 


$\begin{array}{ll}\mathrm{r} & \text { Receiver } \\ \mathrm{s} & \text { Sampling } \\ \mathrm{sr} & \text { Source } \\ \text { tot } & \text { Total variable } \\ \mathrm{w} & \text { Power }\end{array}$

\section{Introduction}

As an estimated number of 210 million European people (44\% of the EU25 a population) is regularly exposed to road traffic noise levels exceeding $55 \mathrm{~dB}(\mathrm{~A})$ (the WHO threshold of serious annoyance ${ }^{1}$ ), reduction of road traffic noise in urban areas is of major concern. From road traffic in urban areas, with typical speeds lower than $50 \mathrm{~km} / \mathrm{h}$, the main noise source is due to the internal combustion engine, see e.g. Jonasson et $a l .^{2}$ This noise is radiated by structural vibrations of the vehicle and the intake and exhaust pipes, which is called shell noise. Also, noise is radiated from the orifices of the intake and exhaust pipe systems. This latter source is of concern here.

In recent EU-funded projects, outdoor sound propagation models and emission models for European road vehicles have been presented in order to generate the obligatory noise maps of EU member states' larger agglomerations. $^{2,3}$ In the road traffic emission model, emphasis is laid towards the development of the complete definition of the noise emission of the average European road vehicle in 1/3-octave bands. A simple model was constructed describing the sound power from tyre-road and propulsion noise, which is a function of the vehicle speed and type. Configuration dependent corrections as for the asphalt type can also be made. In the propagation model, the vehicles are modelled by point sources with two or three source heights, which determine the interference effects with the ground surface. The point sources should be assigned both horizontal and vertical directivity if required. ${ }^{2}$ A vertical, source type independent, directivity was determined with the main purpose to take into account car body screening. A horizontal directivity function was proposed for tyre-road noise. For propulsion noise, a directivity is included only for heavy vehicles and a high propulsion noise source position. All these emission and directivity models are derived from laboratory, test-site and on-site measurements. As a consequence, the separate effects that determine the directivity of road traffic noise sources have not been distinguished systematically. More specifically regarding exhaust pipe noise, the radiated noise is both affected by the thermal and aerodynamical characteristics of nonuniform jet flow leaving the exhaust and by its surrounding geometry. Their separate effects have not been revealed by the measurements, carried out in the projects mentioned above. Also, studies to understand and reduce propulsion noise are ongoing, and computational methods able to predict propulsion noise radiation are very useful in addition to measurements.

Effects of the non-uniform jet flow on free field sound radiation from pipes has been subject to several studies, see e.g. references. ${ }^{4-6}$ There is however a lack of numerical studies to the combined effect of surrounding geometry and jet flow on the power and directivity of the radiated sound field from exhaust pipes. This paper focuses on such a numerical study. The paper elaborates on an approach adopted in recent work, ${ }^{8}$ in which the mean flow properties in and behind the exhaust pipe are calculated by solving the Reynolds averaged Navier-Stokes equations (RANS). The linearized Euler equations (LEE) are then solved using the extended Fourier pseudospectral time-domain method to compute the noise radiated from the exhaust. This Fourier pseudospectral time-domain method is a computationally efficient method to solve the LEE, as only 2 spatial points per acoustic wavelength are required and the extension enables to solve for staircase-type geometries. ${ }^{7}$ This method is in this paper further referred to as PSTD. Whereas the work in ${ }^{8}$ focussed on sound propagation in the presence of a non-uniform jet flow and uniform temperature, in free field and over a rigid ground surface, the current paper extends this work by considering the effect of a cold and hot jet and of a more complex geometrical configuration. Furthermore, the efficiency of the PSTD method is increased by utilizing a multi-domain PSTD approach as presented in. ${ }^{9}$ This implies that a fine mesh is used in the region where high gradients of the ambient variables are occurring, i.e. near the pipe orifice, and a coarser mesh is used for the bulk of the domain.

The paper is organized as follows. In section II, the studied configuration of the exhaust pipe in the environment of a ground surface and an automotive body, as well as the jet flow configurations, are described.

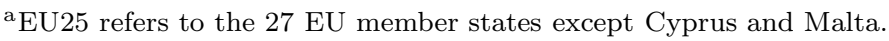




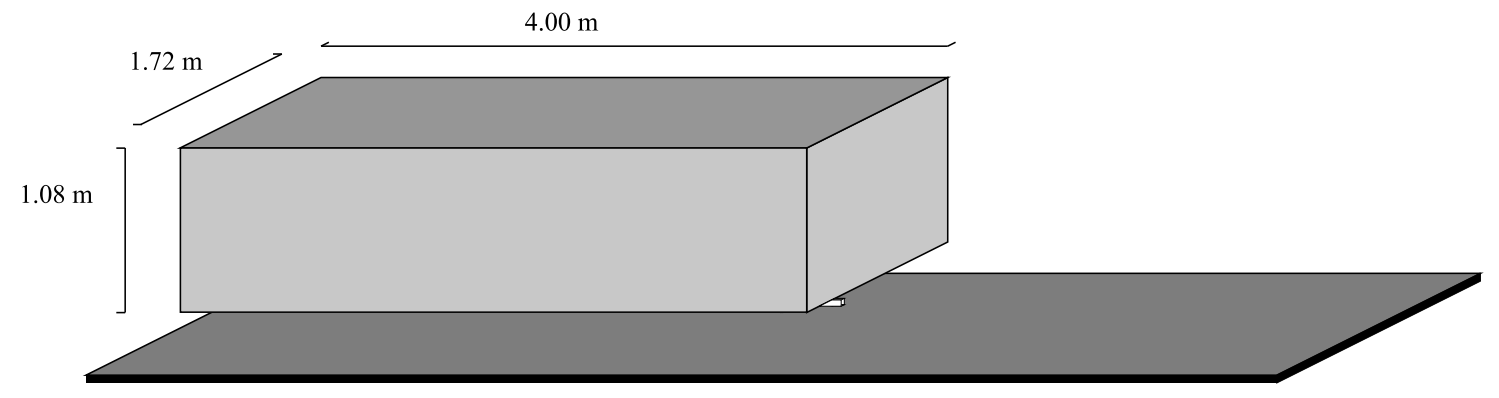

a)

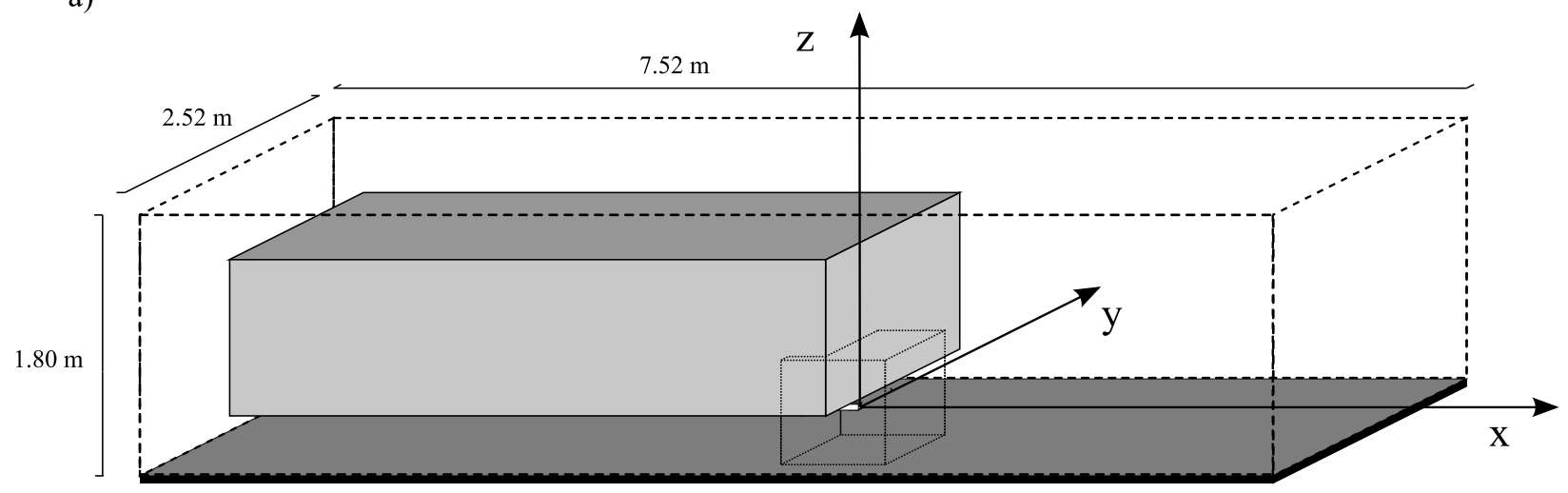

\section{b)} $0.36 \mathrm{~m}]$

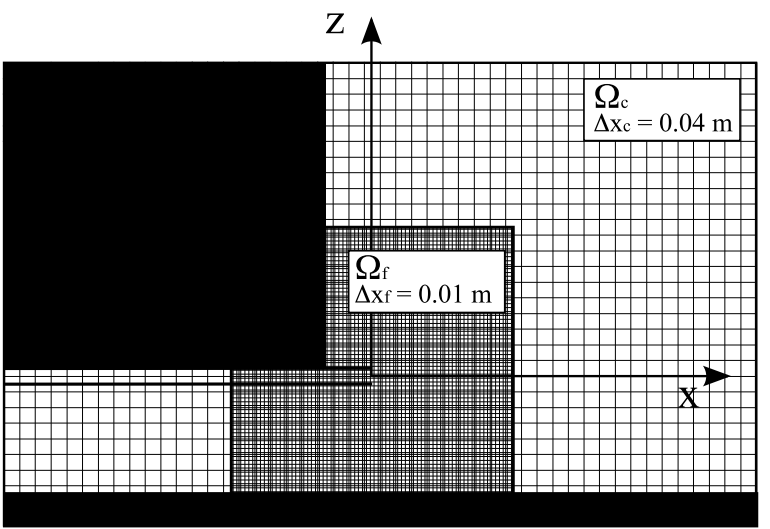

c) $\mathrm{y}=0 \mathrm{~m}$

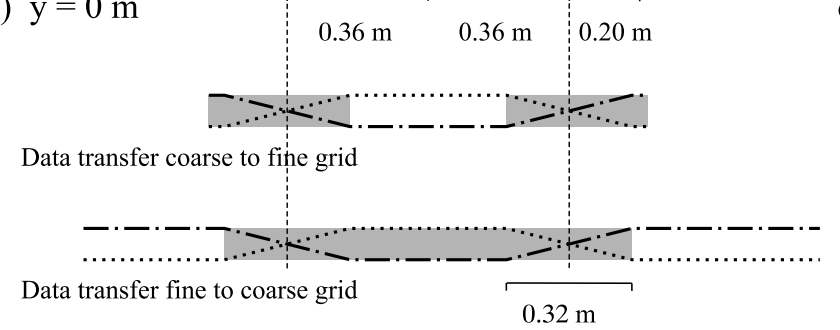

\section{$\mathrm{Z} A$}

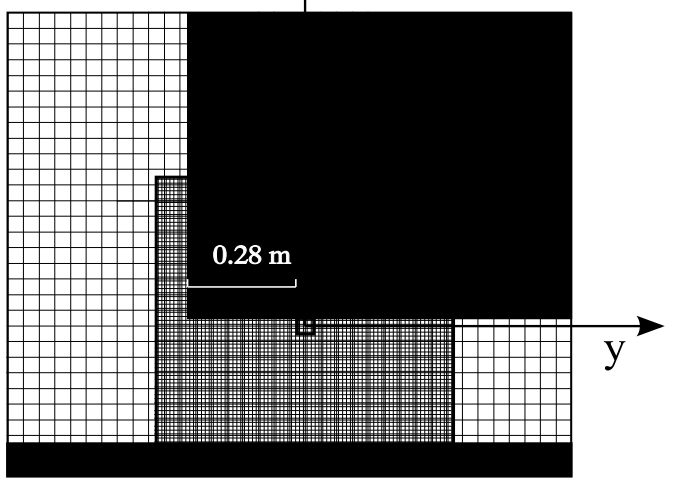

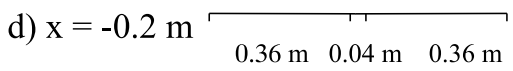

$\begin{array}{ll} & \text { Data transfer region } \\ \ldots . & \text { Multiplication window fine grid values } \\ \ldots . & \text { Multiplication window coarse grid value }\end{array}$

Figure 1. a) Studied configuration of an exhaust pipe over a rigid ground surface in the presence of a rigid body; b) Discretization volumes of the multi domain extended Fourier PSTD method covering the computational domain: the small volume contains the fine PSTD mesh and the large volume contains the coarse PSTD mesh. Receiver positions are located outside the largest volume and computed by integrating the solution at the outer surfaces of the larger volume; c) Part of the cross section at $y=0 \mathbf{m}$ with coarse and fine grids illustrated. Data transfer methodology is depicted below the cross section. d) Part of the cross section at $x=-0.2 \mathrm{~m}$. 
The equations that govern sound radiation from an automotive exhaust pipe can be found in section III as well as the outline of the computational approach of solving those equations. Also, the essentials of the utilized multi domain methodology are briefly addressed in this section. Results from the numerical study are presented in section IV. First, the applicability of the PSTD method to compute sound propagation in the presence of a hot jet flow is shown by comparing PSTD results with results from a Discontinuous Galerkin (DG) method. Afterwards, the results for the proposed configuration are presented and analyzed. Finally, conclusions can be found in section V. It needs to be stressed that the current study is a preliminary study on the combined effects of flow and surrounding geometry. Plans of continued work are also addressed in the final section.

\section{Studied configuration}

The geometry surrounding an automotive exhaust pipe typically consists of a ground surface and the automotive body. In this initial study, this geometry is simplified in a three dimensional (3-D) configuration: the ground surface is represented by a rigid horizontal plane below the pipe and the automotive body by a rigid cuboid on top of the tailpipe, see figure 1(a). The square exhaust pipe measures $40 \mathrm{~mm} \times 40 \mathrm{~mm} \times 4120$ $\mathrm{mm}$ in respectively the $z, y$ and $x$ direction and is located $0.28 \mathrm{~m}$ above the ground surface, placed off center in the width of the body and its exit is located at $0.12 \mathrm{~m}$ from the end of the body. The cuboid measures 1.08 $\mathrm{m} \times 1.72 \times 4.00 \mathrm{~m}$ in respectively the $z, y$ and $x$ direction, which is in accordance with realistic automotive geometries. The automotive body is extremely simplified with the aim to study first order geometrical effects. Computations are made for the configuration of figure 1(a) as well as for the same pipe in the absence of the body and in the absence of both the body and ground surface. For the problem under consideration, an exhaust jet flows through the pipe. The jet has an inflow velocity of $u_{0}=104 \mathrm{~m} / \mathrm{s}$, corresponding to a Mach number of $M=u_{0} / c_{0}=0.3$ and has either an ambient temperature or an inlet temperature of $T_{0}=723 K^{\mathrm{b}}$, further referred to as the cold and hot jet. The hot jet has realistic conditions for an automotive exhaust engine, see e.g. reference. ${ }^{12}$ An incident broadband plane sound wave in the pipe is considered, representing sound being generated in the combustion engine or by upstream flow noise sources. Results are presented in $1 / 3$-octave bands and as exhaust pipe noise has most of its energy in the lower frequency region, i.e. around $100 \mathrm{~Hz}$, the analysis is limited to frequencies of the $2500 \mathrm{~Hz} \mathrm{1/3-octave} \mathrm{band.} \mathrm{All} \mathrm{frequencies} \mathrm{are} \mathrm{below} \mathrm{the}$ cut-on frequency of transversal duct modes. Of interest are the radiated power and directivity. The radiated power level results as presented in section IV are computed as:

$$
\begin{aligned}
L_{w, j} & =10 \log _{10}\left(\frac{\sum_{i=1}^{10} W_{r a d, i j}}{\sum_{i=1}^{10} W_{i n, i j}}\right), \\
W_{r a d, i j} & =\frac{1}{2} \int \mathcal{R}\left[p_{i j} \overline{v_{i j}}\right] \cdot \overrightarrow{\mathbf{n}} \mathrm{d} S_{r}, \\
W_{i n, i j} & =\frac{(1+M)^{2}}{2 \rho_{0} c_{0}} \int\left|p_{i j, i n}\right|^{2} \mathrm{~d} S_{p},
\end{aligned}
$$

with $W_{\text {rad }}$ the radiated power and $W_{i n}$ the power incident in the pipe, $p_{i j, i n}$ the pressure component of the incident sound wave and where $M, \rho_{0}$ and $c_{0}$ are the values in the pipe. The overbar denotes the complex conjugate and the integrals are over $S_{r}$, the receiver planes surrounding the geometry illustrated by the outer volume of figure 1(b) and $S_{p}$, the cross section of the pipe. The indices $j$ and $i$ represent the $1 / 3$-octave band number and the frequencies within the $1 / 3$-octave bands. For the latter, 10 frequencies have been chosen. Isentropic conditions in the pipe are assumed for the computation of $W_{i n}$. Directivity results are defined as the sound pressure level relative to the maximum sound pressure level for a certain $1 / 3$-octave band:

$$
L_{p, j}(R, \phi, \theta)=10 \log _{10}\left(\frac{\sum_{i=1}^{10}\left|p_{j i}(R, \phi, \theta)\right|^{2}}{\max _{\phi, \theta}\left(\sum_{i=1}^{10}\left|p_{j i}(R, \phi, \theta)\right|^{2}\right)}\right),
$$

where $R$ is the fixed distance of the receiver plane, $\phi$ the horizontal angle and $\theta$ the vertical angle, see e.g. figure 3 .

\footnotetext{
${ }^{\mathrm{b}}$ Note that the conditions of $u_{0}=104 \mathrm{~m} / \mathrm{s}$ and $T_{0}=723 \mathrm{~K}$ imply an inlet Mach number of $M=0.19$.
} 


\section{Computational approach}

\section{III.A. Linearized Euler equations (LEE)}

The equations that govern the physical phenomena of noise radiation from the exhaust pipe are the NavierStokes (NS) equations. Effects of molecular viscosity are neglected and all physical variables are decomposed into their ambient values denoted by subscript 0 , and acoustic fluctuations:

$$
\begin{aligned}
\rho_{\mathrm{tot}} & =\rho_{0}+\rho, \\
\mathbf{u}_{\mathrm{tot}} & =\mathbf{u}_{0}+\mathbf{u}, \\
p_{\mathrm{tot}} & =p_{0}+p .
\end{aligned}
$$

As reported e.g. by Davies and Holland, ${ }^{11}$ the amplitudes of the acoustic fluctuations in an automotive exhaust pipe may reach the non-linear regime, yet with peak to mean pressure amplitudes up to 1.1, the error introduced by linearizing with respect to the acoustic variables is expected to be small. The linearized Euler equations (LEE) in non-conservative form are obtained after subtracting the equations for the ambient variables,

$$
\begin{aligned}
\frac{\partial \mathbf{q}}{\partial t}+ & \mathbf{A}_{j} \frac{\partial \mathbf{q}}{\partial j}+\mathbf{C q}=\mathbf{q}_{s r}, \\
\mathbf{A}_{j}= & {\left[\begin{array}{ccccc}
u_{0, j} & \rho_{0} \delta_{x, j} & \rho_{0} \delta_{y, j} & \rho_{0} \delta_{z, j} & 0 \\
0 & u_{0, j} & 0 & 0 & \frac{\delta_{x, j}}{\rho_{0}} \\
0 & 0 & u_{0, j} & 0 & \frac{\delta_{y, j}}{\rho_{0}} \\
0 & 0 & 0 & u_{0, j} & \frac{\delta_{z, j}}{\rho_{0}} \\
0 & \gamma p_{0} \delta_{x, j} & \gamma p_{0} \delta_{y, j} & \gamma p_{0} \delta_{z, j} & u_{0, j}
\end{array}\right], } \\
\mathbf{C}= & {\left[\begin{array}{ccccc}
\frac{\partial u_{0, j}}{\partial j} & \frac{\partial \rho_{0}}{\partial x} & \frac{\partial \rho_{0}}{\partial y} & \frac{\partial \rho_{0}}{\partial z} & 0 \\
\frac{u_{0, j}}{\rho_{0}} \frac{\partial u_{0, x}}{\partial j} & \frac{\partial u_{0, x}}{\partial x}+D & \frac{\partial u_{0, x}}{\partial y} & \frac{\partial u_{0, x}}{\partial z} & 0 \\
\frac{u_{0, j}}{\rho_{0}} \frac{\partial u_{0, y}}{\partial j} & \frac{\partial u_{0, y}}{\partial x} & \frac{\partial u_{0, y}}{\partial y}+D & \frac{\partial u_{0, y}}{\partial z} & 0 \\
\frac{u_{0, j}}{\rho_{0}} \frac{\partial u_{0, z}}{\partial j} & \frac{\partial u_{0, z}}{\partial x} & \frac{\partial u_{0, z}}{\partial y} & \frac{\partial u_{0, z}}{\partial z}+D & 0 \\
0 & \frac{\partial p_{0}}{\partial x} & \frac{\partial p_{0}}{\partial y} & \frac{\partial p_{0}}{\partial z} & \gamma \frac{\partial u_{0, j}}{\partial j}
\end{array}\right], } \\
D= & \frac{\partial u_{0, j}}{\partial j}+\frac{u_{0, j}}{\rho_{0}} \frac{\partial \rho_{0}}{\partial j},
\end{aligned}
$$

with $\mathbf{q}=\left[\rho, u_{x}, u_{y}, u_{z}, p\right]^{T}, \mathbf{q}_{s r}$ the source vector, index $j$ equal to $x, y z, \gamma$ the heat capacity ratio and $\delta$ the Kronecker delta function.

\section{III.B. Multi domain extended Fourier pseudospectral time-domain method (PSTD)}

Equations (4) are solved by the PSTD method on an orthogonal equidistant grid, with velocity nodes staggered with the pressure and density nodes. The spatial derivatives are computed using the extended Fourier pseudospectral method, which relies on an eigenfunction expansion of the LEE for a medium at rest and assuming isentropy. ${ }^{7}$ Since FFTs are used to calculate derivatives, only two spatial points per wavelength are needed with this method. The following source vector is used:

$$
\begin{aligned}
& \mathbf{q}_{s r}(\mathbf{x}, t)=\left[\begin{array}{c}
\frac{\delta\left(x-x_{s r}\right)}{c_{0}^{2}} \sin \left(2 \pi f_{s r} t\right) e^{-b\left(t-t_{s r}\right)^{2}} \\
0 \\
0 \\
0 \\
\mathbf{q}_{s r}(\mathbf{x}, t)=0 \\
\delta\left(x-x_{s r}\right) \sin \left(2 \pi f_{s r} t\right) e^{-b\left(t-t_{s r}\right)^{2}}
\end{array}\right] \text { for } \quad y, z<|a|,
\end{aligned}
$$

is used with $a$ the half pipe width, $\mathbf{x}=[x, y, z], x_{s r}=-1.52 \mathrm{~m}$ the source location, $t_{s r}=0.001 \mathrm{~s}$ is the central time, $b=3 f_{s}^{2} / 16 \mathrm{~s}^{2}$ a constant determining the spectral bandwidth and $\delta\left(x-x_{s r}\right)$ the Dirac delta 
function. $f_{s r}=f_{s} / 8 \mathrm{~Hz}$ the central frequency with $f_{s}=c_{0} / \Delta x$ the sample frequency corresponding to the spatial discretization. The source conditions are assumed to be adiabatic as the ambient pressure and density are set constant in the tailpipe. At every discrete time step, the equations are marched in time by a low-storage optimized 6-stage Runge-Kutta method. The box surrounding the studied configuration in figure 1(b) illustrates the computational boundaries of the PSTD method. Outside these boundaries, a PML is included to obtain non-reflective conditions as in reference. ${ }^{7}$ Also, the non-radiating end of the exhaust pipe at $x=-4.00 \mathrm{~m}$ is terminated by a PML.

The mean flow variables of equations (4) are obtained by solving the RANS equations using commercial software. As shown in, ${ }^{8}$ this approach is suitable for jet flows. As the computational grid for the RANS solution differs from the orthogonal PSTD grid, a mapping of the RANS results needs to be applied. In this paper, the mean variables are filtered to the PSTD grid by applying a rectangular filter:

$$
\mathbf{q}_{0}(\mathbf{x})=\left[\begin{array}{ccc}
\frac{\sum_{i} \mathbf{q}_{0, R A N S}\left(\mathbf{x}_{i}\right) S\left(\mathbf{x}_{i}\right)}{\sum_{i} S\left(\mathbf{x}_{i}\right)} & \text { for } & \left|\mathbf{x}_{i}-\mathbf{x}\right| \leq 2 a / m \\
0 & \text { for } & \left|\mathbf{x}_{i}-\mathbf{x}\right|>2 a / m
\end{array}\right.
$$

with $i$ the index running over all nodes of the RANS solution mesh, $S_{i}$ the cell volume belonging to node $i$ and $m$ determining the cut-off wavenumber of the filtering operation by $k_{c}=2 a \pi / \mathrm{m} \mathrm{m}^{-1}$. A low $k_{c}$ number will spatially smooth the ambient flow components, thereby promoting stability of the physical as well as numerical solution to the LEE, but also suppresses some relevant physical processes. A too high $k_{c}$ number could, on the other hand, lead to spurious oscillations in the numerical solution to the LEE as small wave numbers which are not resolved by the discretization of the used PSTD methodology, are present in the solution. A number of $m=8$ is chosen as a trade-off for all calculations of this paper.

For the numerical solution of the LEE, the required grid spacing near the tailpipe exit is determined by the dimensions of the local geometry and mean flow gradients and is finer than needed for resolving the smallest acoustical wavelength of interest in the bulk of the domain, see e.g. ${ }^{8}$ As a result, an orthogonal equidistant PSTD mesh will over resolve most of the domain, it is attractive to apply a multi domain approach, i.e. a fine grid in the vicinity of the tailpipe and a coarser grid elsewhere. For this reason, the multi domain PSTD methodology that has recently been proposed in $^{9}$ is utilized, and is summarized here.

The total domain is composed by the coarse and fine grids without their overlapping areas, depicted by the large and small box in figure 1(b). Coarse grid pressure and densities are staggered with their fine grid counterparts in all directions. The coarse sub-domain $\Omega_{c}$ covers the total domain thereby extending outside the coarse-fine grid interface into the fine grid area; see figure $1(\mathrm{c}, \mathrm{d})$. In the extended region, data transfer takes place from the fine to the coarse grid. The fine sub-domain $\Omega_{f}$ extends outside the coarse-fine grid interface into the coarse grid. In the extended region, data transfer takes place from the coarse to the fine grid. In the outermost region of the fine grid, values are multiplied by a super-Gaussian window function to obtain periodicity in $\Omega_{f}$. The coarse grid $\Omega_{c}$ is discretized with equidistant spacing $\Delta x_{c}$, the fine grid $\Omega_{f}$ with equidistant spacing $\Delta x_{f}=\Delta x_{c} / d f$ with $d f$ the discretization factor being an integer. The discrete time step of the coarse grid is $\Delta t_{c}=\frac{\Delta x_{c}}{2 c_{0}}$, as in, ${ }^{7}$ and the fine grid has time step $\Delta t_{f}=\frac{\Delta t_{c}}{d f}$. During one time iteration $\Delta t_{c}$, the fine grid results are iterated $d f$ times without data transfer with the coarse grid at intermediate time steps. After each $\Delta t_{c}$, data transfer takes place from coarse to fine grid and vice versa, see figure 1(c). Coarse grid data are spectrally interpolated to the fine grid positions and fine grid data are decimated by factor $d f$ and spectrally interpolated to the coarse grid positions. As the region behind the tailpipe, modelled by the fine grid could lead to smaller wavelengths than resolved by the coarse grid, a low pass filter could be applied to the fine grid prior to decimation.

The multi domain methodology was shown not to introduce significant errors over a single PSTD method yet requires a suitable choice of the dimensions of the data transfer zones. ${ }^{9}$ A super-Gaussian window corresponding to an error of $-40 \mathrm{~dB}$ has been chosen here, along with a width of $0.2 \mathrm{~m}$ outside the subdomain interfaces for the coarse to fine grid data transfer region, see figure 1(c). The current multi-domain application was found to lead to larger errors as in. ${ }^{9}$ These errors arise from a discrepancy of the aliasing error from both grids due to the wave appearing at the tailpipe origin, which acts as a point-like source. To overcome this problem, the data transfer takes place with a linear interpolation, as depicted in figure 1(c). As such, continuity of the variable values is promoted. 


\section{III.C. Kirchhoff-Helmholtz integral equation (KHIE)}

To study the effect of geometry and non-uniform mean flow and temperature on the directivity, far field result are desired. The computational approach is spatially limited by its amount of grid points. When assuming a non-moving medium and a rigid ground surface outside the computational domain of figure 1(b), the KHIE can be solved to compute the solution at an arbitrary receiver position outside the PSTD domain:

$$
\begin{aligned}
p_{j i}\left(\mathbf{x}_{r}\right) & =\int_{S_{c}}\left(G_{j i}\left(\mathbf{x}_{r} \mid \mathbf{x}_{s r}\right) \frac{\partial p_{j i}\left(\mathbf{x}_{s r}\right)}{\partial n}-p_{j i}\left(\mathbf{x}_{s r}\right) \frac{\partial G_{j i}\left(\mathbf{x}_{r} \mid \mathbf{x}_{s r}\right)}{\partial n}\right) \mathrm{d} \mathbf{x}_{s r} \\
G_{j i}\left(\mathbf{x}_{r} \mid \mathbf{x}_{s r}\right) & =\frac{e^{j k_{j i} R}}{4 \pi R}+\frac{e^{j k_{j i} R^{\prime}}}{4 \pi R^{\prime}} \\
R & =\left|\mathbf{x}_{r}-\mathbf{x}_{s r}\right| \\
R^{\prime} & =\left|\mathbf{x}_{r}-\mathbf{x}_{s r}^{\prime}\right| \\
\mathbf{x}_{s r} & =\left[x_{s r}, y_{s r}, z_{s r}\right] \\
\mathbf{x}_{s r}^{\prime} & =\left[x_{s r}, y_{s r},-z_{s r}-0.6\right]
\end{aligned}
$$

with $p_{j i}\left(\mathbf{x}_{s r}\right)$ and $\frac{\partial p_{j i}\left(\mathbf{x}_{s r}\right)}{\partial n}$ the frequency components $i$ of $1 / 3$-octave band $j$ obtained after Fourier transforming pressure and normal velocity results from the PSTD method with positions at the integration surfaces $S_{c}$, located at the outer volume of the computational domain as depicted in figure 1(b). $R$ and $R^{\prime}$ are respectively the distance from the receiver position to a position at $S_{c}$ and to the image of the position at $S_{c}$ with the ground surface. As equation (7) is evaluated by numerical quadrature, higher spatial resolution as in the PSTD grid is necessary to accurately compute the highest $1 / 3$-octave band of interest. A resolution of 17 points per wavelength for $2500 \mathrm{~Hz}$ was used, a grid spacing of $\Delta x_{c} / 5=0.008 \mathrm{~m}$, and was found to lead to a maximum error of $0.4 \mathrm{~dB}$ for an analytical case. This resolution was obtained by spectrally interpolating the PSTD data at the surfaces $S_{c}$ prior to application of the Fourier transforms.

\section{Results}

\section{IV.A. Validity of the PSTD approach for hot flows}

In a previous study, ${ }^{8}$ a good agreement was obtained between results from the PSTD approach and results from an analytical model for sound radiation from a pipe with a jet flow with Mach number $\mathrm{M}=0.3$ and uniform temperature. As the influence of a hot flow on the radiated field is considered in this paper as well, the PSTD solution to equations (4) in the presence of a hot flow is for validation compared with results from a Discontinuous Galerkin (DG) solution of the LEE. The DG is a high-accurate numerical method to solve the LEE and its details can be found e.g. in reference. ${ }^{13}$ For this validation case, a 2-D configuration of the exhaust pipe in free field is chosen, depicted by figure 2(c). PSTD implementations are tested with a single grid and a multi domain grid. The DG mesh consists of triangles. In the DG method, a polynomial order of 5 has been used and inter-element fluxes are computed making use of the method of characteristics. From the RANS solution, the horizontal velocity component $u_{x}$ and the temperature $T$ are shown in figure $2(\mathrm{a}, \mathrm{b})$. Figures 2(d,e) show the angular dependent sound pressure relative to the incident pressure level, i.e.:

$$
L_{p, i n}(r, \theta)=10 \log _{10}\left(\frac{|p(R, \theta)|^{2}}{\left|p\left(-0.4, \theta_{0}\right)\right|^{2}}\right),
$$

is shown for two frequencies and $\theta_{0}=180 \mathrm{deg}$. In equation (8), the pressures are expressed in the polar coordinate system of figure 2 , with origin at the tailpipe exit. The results, shown in figures $2(\mathrm{e}, \mathrm{f})$, display a good agreement between the DG and extended Fourier PSTD methods, showing the ability of the extended Fourier PSTD method to compute the effect of a hot jet on the radiated sound from an exhaust pipe. Results from the multi domain PSTD collapse for most of the angles with the single domain PSTD results apart from the low angles in the $2000 \mathrm{~Hz}$ case. As no numerical or physical instabilities were detected, no filtering in the fine grid was needed for this 2 -D validation case. 


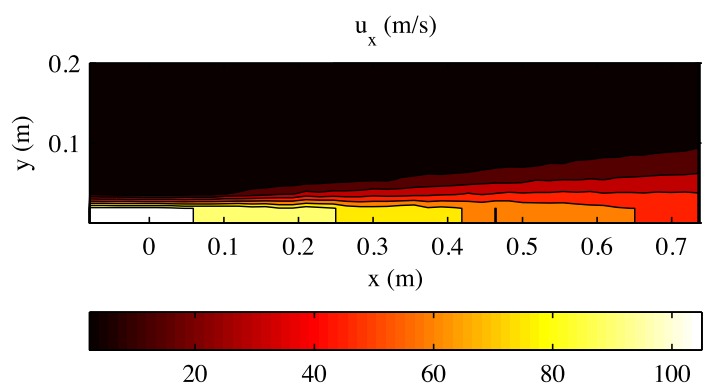

a)

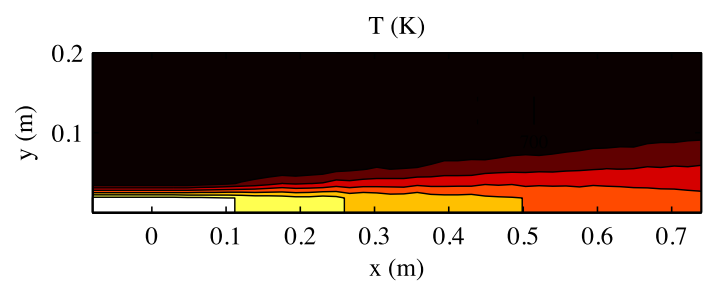

b)

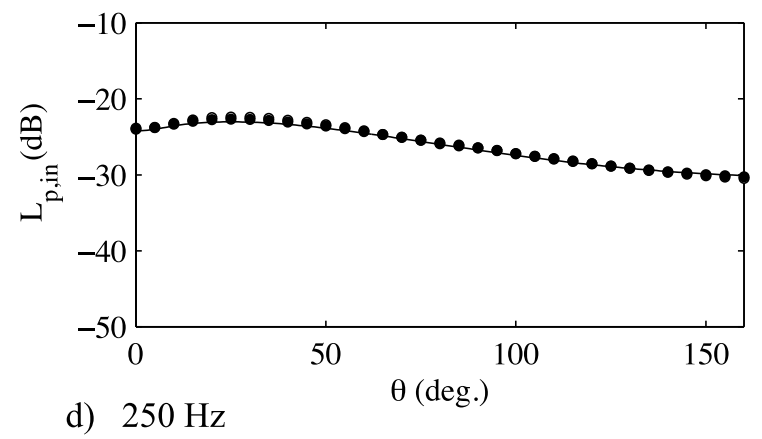

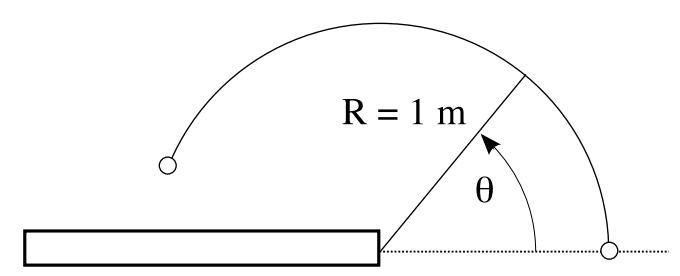

c)

•..... PSTD, single grid

0000000 PSTD, multi domain grid DG

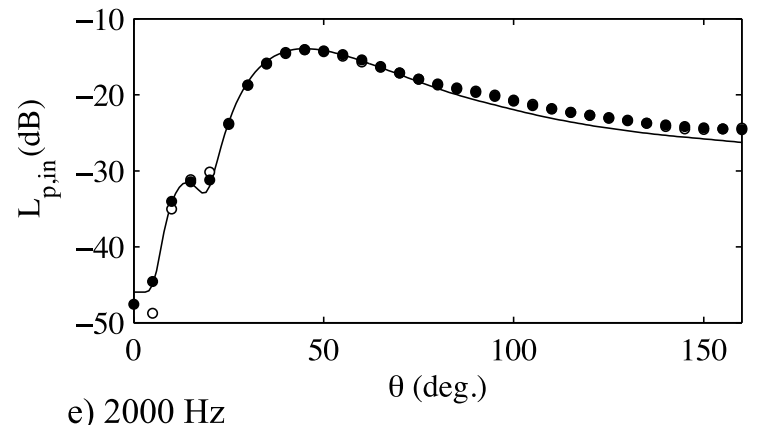

e) $2000 \mathrm{~Hz}$

Figure 2. Radiated levels in free field relative to the level incident in the tailpipe, $L_{\mathrm{p}}$, in , in the presence of a hot jet. a,b) Mean horizontal velocity component and temperature fields used in the computations; c) 2-D Free field configuration studied; d,e) Results from DG and extended Fourier PSTD methods as a function of the radiation angle. 

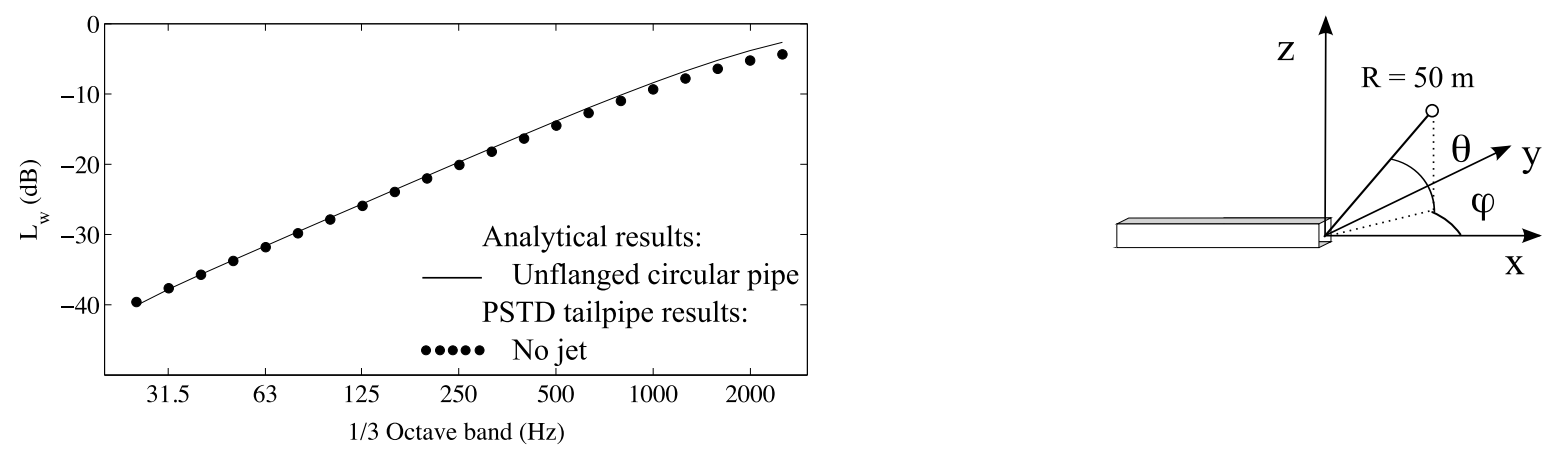

a) $\mathrm{Lw}_{w}$

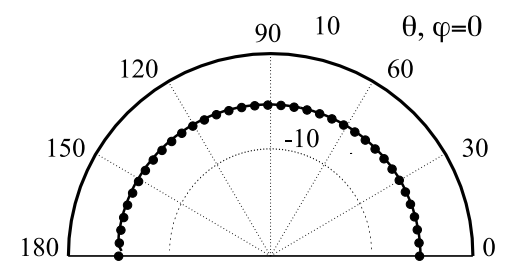

b) $\mathrm{L}_{\mathrm{p}}, 63 \mathrm{~Hz}$

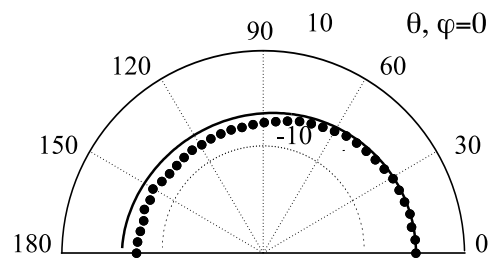

c) $\mathrm{L}_{\mathrm{p}}, 2000 \mathrm{~Hz}$

Figure 3. Multi domain extended Fourier PSTD results for the configuration with tailpipe in free field. Cases without jet, with a cold jet and a hot jet are computed. a) $L_{w}$ : Sound power level relative to the incident power in the exhaust pipe. b-g) Directivity plots in the $z=0$ plane for the $63 \mathrm{~Hz}$ and $2000 \mathrm{~Hz} \mathrm{1/3-octave} \mathrm{bands.}$

\section{IV.B. Effect of geometry and flow on directivity and power of the radiated sound field}

All results in this section are computed by the PSTD method using the multi domain approach. A zero phase low pass filter with zero dissipation up to a chosen cut-off wave number of $k_{c}=\pi /(4 a) \mathrm{m}^{-1}$ and a Gaussian shaped decay beyond that has been applied to the fine grid results to avoid instabilities, see reference. ${ }^{8}$ The results are presented for the three configurations in figures 3,4 and 5 . In the absence of the jet, $L_{w}$ is computed and presented in subfigures (a). For the configuration in free field, the radiated power linearly increases with frequency, see figure 3(a). Analytical results from ${ }^{14}$ for the radiated power of an unflanged circular pipe with the same cross section as the rectangular pipe of the current study, i.e. a radius of $\sqrt{a^{2} / \pi}$, are plotted in the same subfigure. The results are in good agreement. The slight deviations at the highest frequencies could be an effect of the applied filter in the multi-domain implementation or by the fact that the pipe under study is not circular. For the lower frequencies in the configuration with the ground surface, the radiated power is higher than for the free field case, see figure 4(a). The low frequency results reach the solution of a pipe with twice the cross section, as depicted by an analytical calculation. This constitutes the solution for the whole frequency range with the pipe would be situated at the ground surface. The presence of the body further increases the radiated power, see figure 5(a). The analytical result, plotted by the dashed line, represents a flanged circular pipe orifice with twice the physical cross section. The surrounding geometry thus causes an increase of about $6 \mathrm{~dB}$.

Directivity results are also plotted in figures 3,4 and 5. The upper halve of the polar plots displays the directivity in the $(x, z)$ plane at $y=0 \mathrm{~m}$, and the lower half the directivity in the $(x, y)$ plane at $z=0 \mathrm{~m}$ for the non free field cases. The receiver positions are all at a distance of $50 \mathrm{~m}$ from the orifice of the pipe. Two $1 / 3$-octave bands are selected for the directivity plots. For the free field case, the $63 \mathrm{~Hz} 1 / 3$-octave band almost shows an omnidirectional pattern. The ground surface does not significantly change the directivity too much, but the automotive body does. A shielding effect is noticed behind the body and some interference effect occur above it. The $2000 \mathrm{~Hz}$ band is significantly more directional for the free field case with highest values behind the pipe exit. Differences with analytical results as computed according to reference ${ }^{15}$ could have the same nature as those discussed from the sound power results. In the presence of the ground surface, interference effects are clearly visible. The analytical directivity of a point source over a ground surface matches the computed interference pattern. The automotive body strongly influences the directivity with high shielding behind the body. 

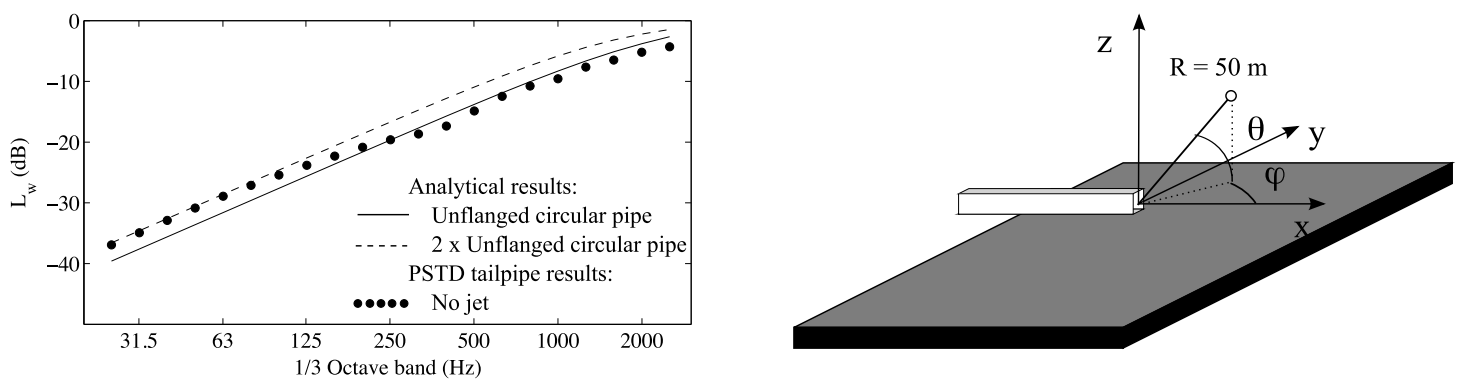

a) $\mathrm{Lw}$
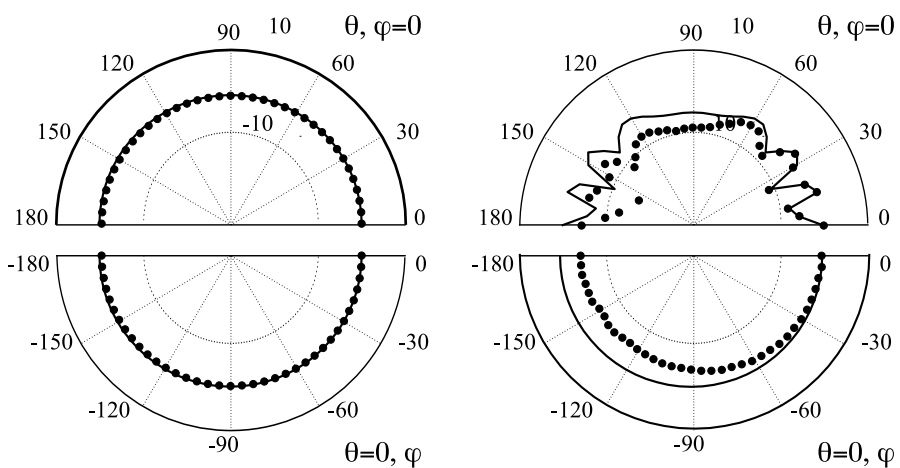

Point source above rigid ground, no jet PSTD tailpipe results:

$\bullet \bullet \quad$ No jet

b) $\mathrm{L}_{\mathrm{p}}, 63 \mathrm{~Hz}$

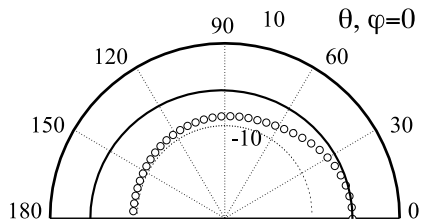

c) $\mathrm{L}_{p}, 2000 \mathrm{~Hz}$

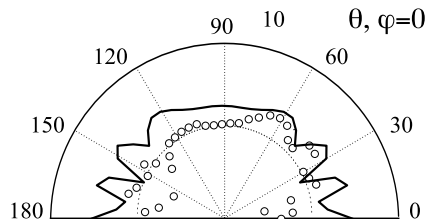

Analytical results:

Point source above rigid ground, no jet PSTD tailpipe results:
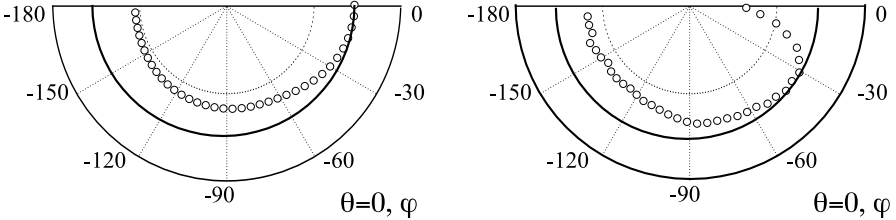

00000 Cold jet, $M=0.3, \mathrm{~T}_{0}=293 \mathrm{~K}$

d) $\mathrm{Lp}, 63 \mathrm{~Hz}$

e) Lp , $2000 \mathrm{~Hz}$
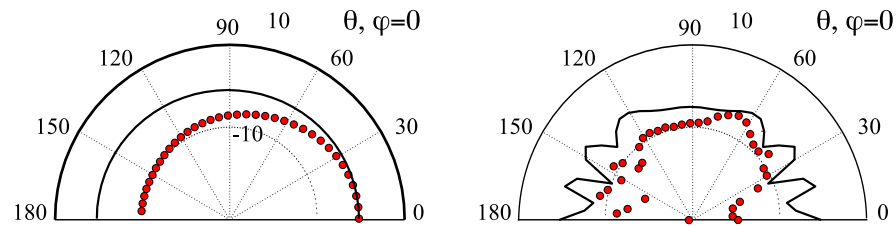

Analytical results:

Point source above rigid ground, no jet PSTD tailpipe results:

Hot jet, $\mathrm{M}=0.19, \mathrm{~T}_{0}=723 \mathrm{~K}$

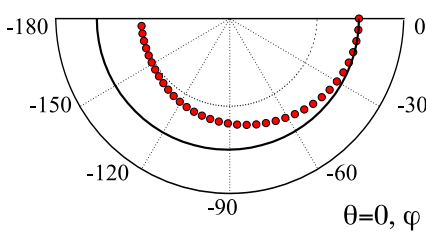

f) $\mathrm{Lp}, 63 \mathrm{~Hz}$

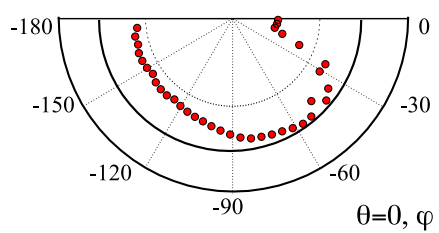

g) $\mathrm{Lp}, 2000 \mathrm{~Hz}$

Figure 4. Multi domain extended Fourier PSTD results for the configuration with tailpipe and ground surface. Cases without jet, with a cold jet and a hot jet are computed. a) $L_{w}$ : Sound power level relative to the incident power in the exhaust pipe. b-g) Directivity plots in the $z=0$ and $y=0$ plane for the $63 \mathrm{~Hz}$ and $2000 \mathrm{~Hz} 1 / 3-0 c t a v e \mathrm{bands}$. 

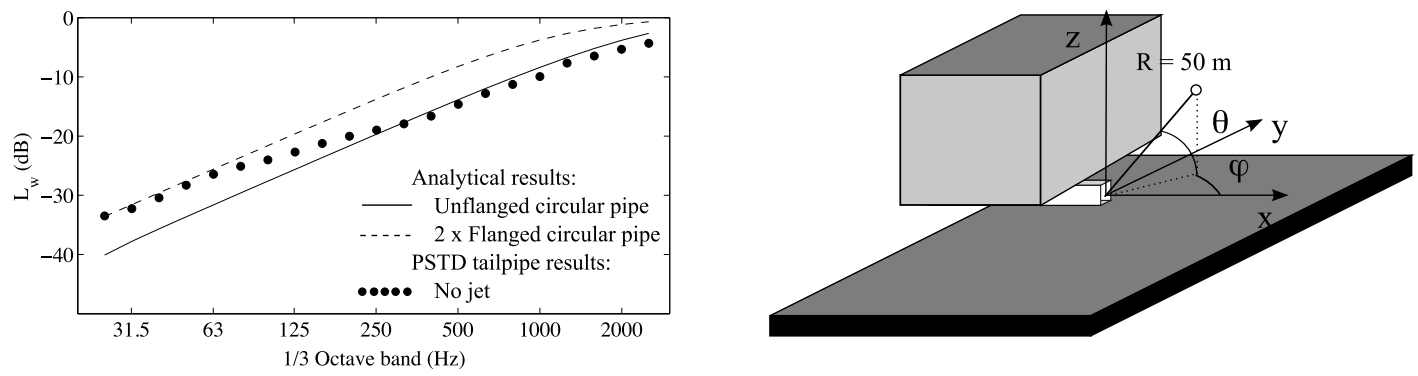

a) $\mathrm{Lw}_{w}$
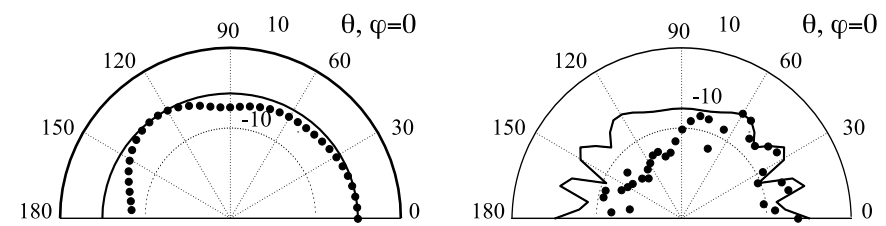

Analytical results:

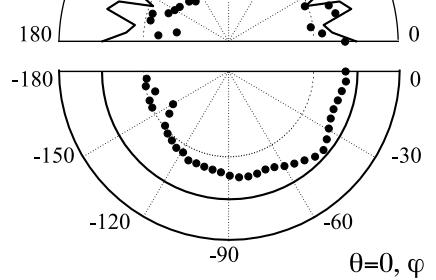

— Point source above rigid ground, no jet PSTD tailpipe results:

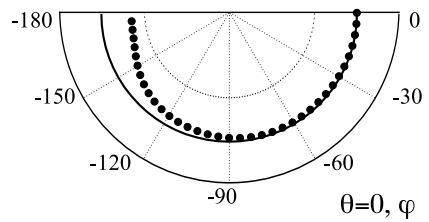

•.... No jet

b) $\mathrm{L}_{p}, 63 \mathrm{~Hz}$

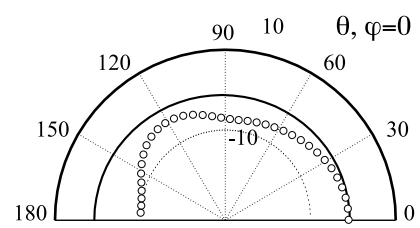

c) $\mathrm{Lp}_{p}, 2000 \mathrm{~Hz}$
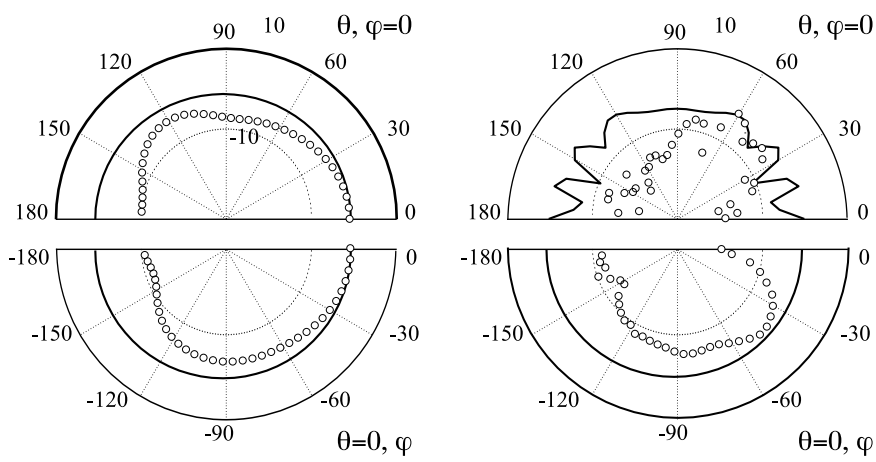
Analytical results:
Point source above rigid ground, no jet
PSTD tailpipe results:

00000 Cold jet, $\mathrm{M}=0.3, \mathrm{~T}_{0}=293 \mathrm{~K}$

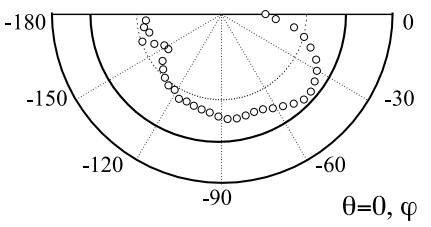

e) $\mathrm{Lp}, 2000 \mathrm{~Hz}$
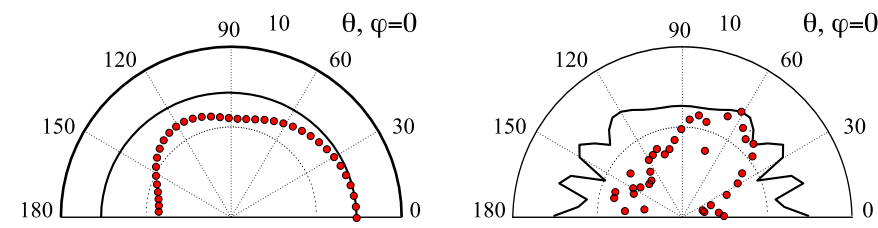
Analytical results:
Point source above rigid ground, no jet PSTD tailpipe results:

$\bullet$ Hot jet, $\mathrm{M}=0.19, \mathrm{~T}_{0}=723 \mathrm{~K}$
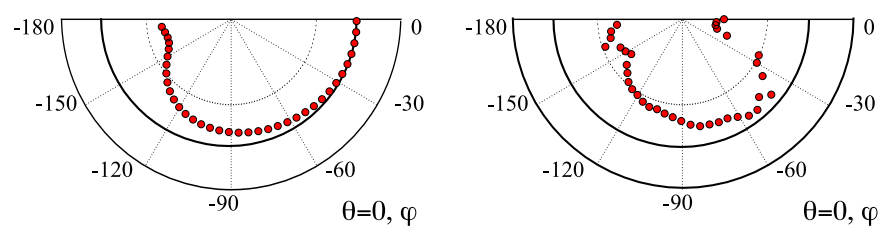$$
\text { . }
$$

f) $\mathrm{Lp}, 63 \mathrm{~Hz}$

g) Lp , $2000 \mathrm{~Hz}$

Figure 5. Multi domain extended Fourier PSTD results for the configuration with tailpipe, ground surface and body. Cases without jet, with a cold jet and a hot jet are computed. a) $L_{w}$ : Sound power level relative to the incident power in the exhaust pipe. b-g) Directivity plots in the $z=0$ and $y=0$ plane for the $63 \mathrm{~Hz}$ and $2000 \mathrm{~Hz} 1 / 3-0 c t a v e \mathrm{bands}$. 


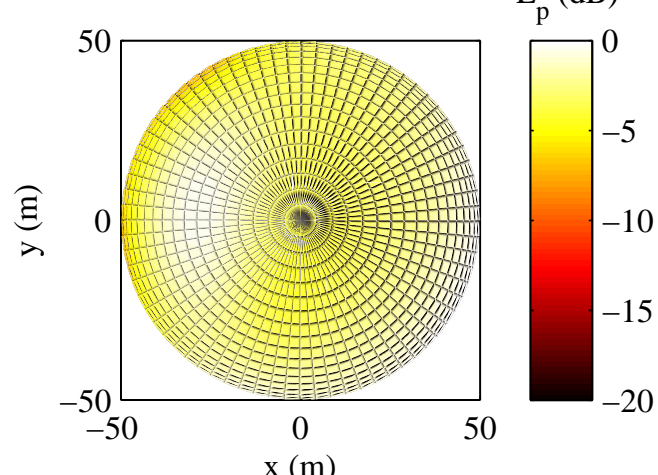

a) No Jet, $63 \mathrm{~Hz}$

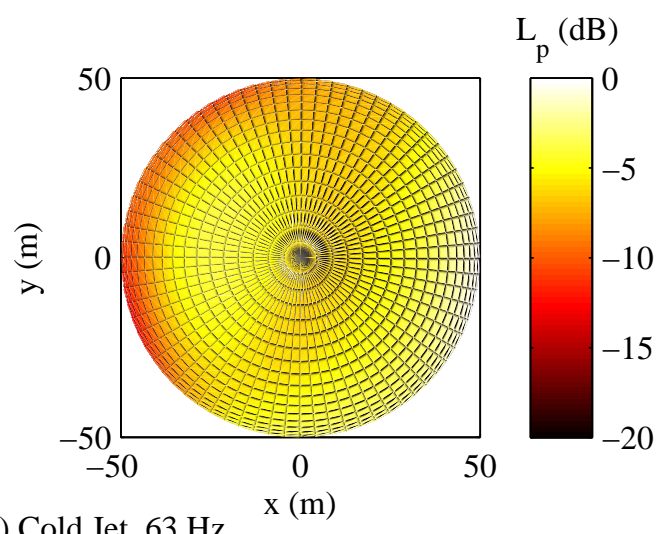

c) Cold Jet, $63 \mathrm{~Hz}$

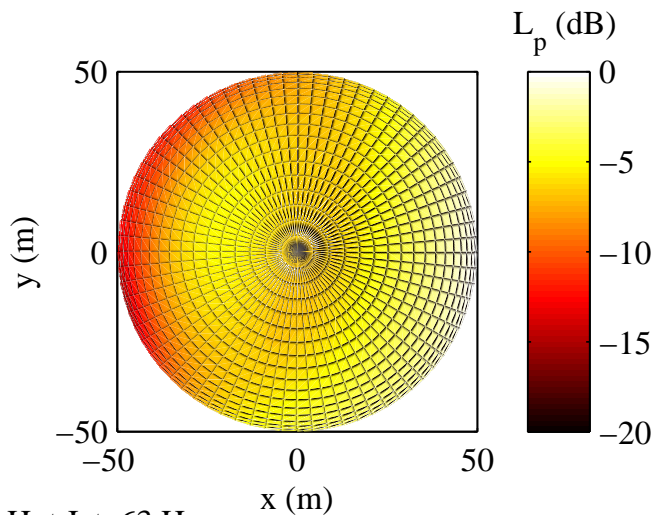

e) Hot Jet, $63 \mathrm{~Hz}$

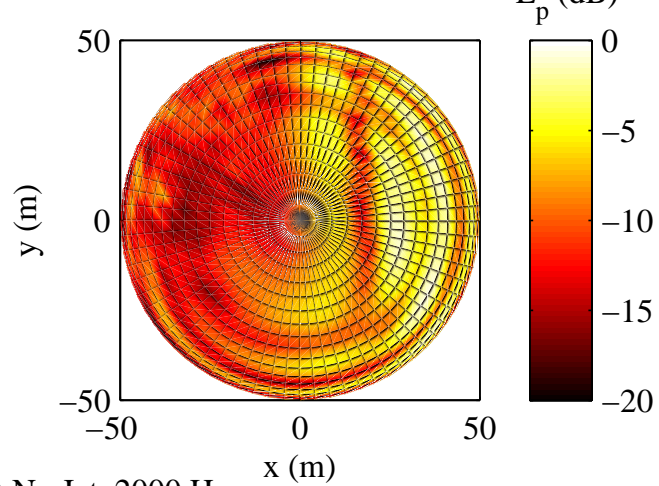

b) No Jet, $2000 \mathrm{~Hz}$

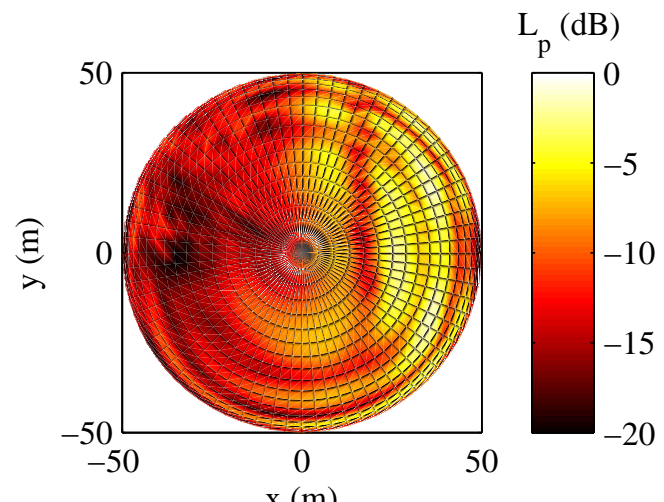

d) Cold Jet, $2000 \mathrm{~Hz}^{\mathrm{x}}(\mathrm{m})$

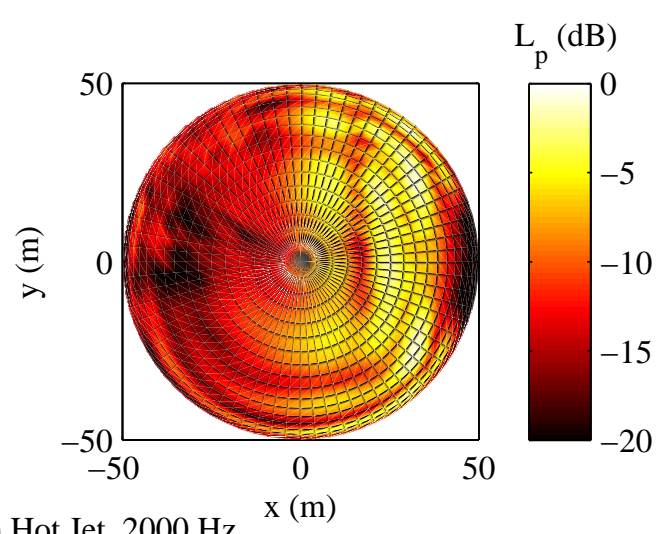

Figure 6. Top view of $L_{p}$. for the configuration with tailpipe, ground surface and body. The case without jet, with the cold jet and with the hot jet are shown.

Figures 6 show the directivity for the ground-body case for all receiver positions in a hemisphere around the configuration by a top view. The result for the $20001 / 3$-octave band in figure (b) clearly shows the ground interferences, an interference with the reflected sound from the body and a the shielding effect. Directivity plots for the cases with cold and hot flow are included for the configurations with ground surface and with ground surface and body, and are shown in subfigures (b) to (g) of figures 4 and5. For the configuration with ground only, the $63 \mathrm{~Hz}$ band has the highest levels in the region behind the pipe and decreases with increasing angle with a reduction up to about $10 \mathrm{~dB}$. The effect is similar for the cold and hot jets. The directivity pattern in the presence of ground, body and flow mimics the results from the same configuration without flow. The additional flow effect is moderate but clearly visible, also in the geometrically screened direction. The 
directivity results with the top view in figure 6 further illustrate that both flow and surroundings determine the directivity. A cone of low sound levels is created behind the pipe for the $2000 \mathrm{~Hz}$ band, and is visible for both cold and hot jets in the configurations with ground and with ground and body. The effect is more pronounced for the hot flow than for the cold flow. For the ground case, it can be noticed that the ground interference pattern is still present, but the amplitudes are shaped by the flow effects. For the ground-body configuration, the main additional effect of the flow on the directivity is in the region behind the exhaust pipe. The geometrically shielded area is much less effected by the flow. These findings can also been seen in the top view plots of figure 6 .

\section{Conclusions and further work}

The present work numerically investigates the effects of exhaust jets with non-uniform mean flow and temperature as well as geometrical effects on the radiated sound field from automotive exhaust pipes. This initial study focusses on a 3-D configuration with a rigid ground surface and an automotive body represented by a rigid cuboid. The RANS equations are solved to obtain the mean flow and temperature field behind the exhaust and a recently proposed multi domain extended Fourier pseudospectral time-domain (PSTD) methodology has been adopted to solve the linearized Euler equations. This approach is computationally efficient, with a fine grid covering the high flow gradients near the exhaust exit and the coarse grid covering the bulk part of the domain. The far field sound pressures are computed by integrating over the solution at the outer boundaries of the PSTD grid. The methodology is successfully verified for a 2-D case of the exhaust pipe in free field and the presence of a hot exhaust jet. For the 3 -D configuration with the ground and body, it has been shown that for the low frequency region where noise from the combustion engine has its main contributions, the geometrical effects lead to a gain of the radiated sound power by $6 \mathrm{~dB}$ compared to a exhaust pipe in free field. The body causes a shielding of the radiated noise, which is more pronounced at higher frequencies. In the absence of the rigid body, the presence of an exhaust flow causes a clear directivity effect, with typically -10 dB lower levels in the backward direction, both for the low and high frequencies. This effect is still visible in the presence of the body, although to a more moderate extent. For the high frequencies, a cone of low noise levels is moreover created behind the exhaust pipe, with similar effects for the configurations with and without body. The described directivity effects are all more pronounced for a hot flow compared to a cold flow.

The work presents initial results and a more systematic study of the dependency of the Mach number and temperature on the directivity and sound power will be carried out. Also, the effects of a ground surface with a realistic impedance and a more representative automotive geometry are of interest. The obtained results could further be compared with the engineering directivity functions as currently used.

\section{Acknowledgments}

This research was supported by a Marie Curie Intra European Fellowship within the 7th European Community Framework Programme.

\section{References}

\footnotetext{
${ }^{1}$ Den Boer, L.C. and Schroten, A.,"Traffic noise reduction in Europe: Health effects, social costs and technical and policy options to reduce road and rail traffic noise", Report: CE Delft, The Netherlands, 2007.

${ }^{2}$ Jonasson, H. et al.,"Source modelling of road vehicles", Deliverable 9 of the HARMONOISE project, report HAR11TR041210-SP10, Borås, Sweden, 2004.

${ }^{3}$ Peeters, B. and van Blokland, G. ,"The Noise Emission Model For European Road Traffic," Deliverable 11 of the IMAGINE project, IMA55TR-060821-MP10, 2007.

${ }^{4}$ Kok, J.C.," Computation of sound radiation from cylindrical ducts with jets using a high-order finite-volume method," Proceedings of the 13th AIAA/CEAS Aeroacoustics Conference, Rome Italy, May 21-23, 2007, AIAA paper 2007-3489.

${ }^{5}$ Zhang, X., Chen, X. and Morfey, C.L.,"Acoustic radiation from a semi-infinite duct with a subsonic jet," International Journal of Aeroacoustics, Vol. 4, No. 1-2, 2005, pp. 169-184.

${ }^{6}$ Manera, J., Schiltz, B., Leneveu, R., Caro, S. and Jacqmot, J.,"Kelvin-Helmholtz instabilities occurring at a nacelle exhaust," Proceedings of the 14th AIAA/CEAS Aeroacoustics Conference, Vancouver, Canada, May 5-7, 2008, AIAA paper 2007-2883.

${ }^{7}$ Hornikx, M., Waxler, R. and Forssén, J.," The extended Fourier pseudospectral time-domain method for atmospheric sound propagation," Journal of the Acoustical Society of America, Vol. 128(4), 2010, pp. 1632-1646.
} 
${ }^{8}$ Hornikx, M., De Roeck, W. and Desmet, W.,"Simplified exhaust pipe noise radiation modelling using the Fourier PSTD method," ISMA2010 Conference, September 20-22, 2010, Leuven, Belgium, paper 488.

${ }^{9}$ Hornikx, M., De Roeck, W. and Desmet, W.," A multi-domain Fourier pseudospectral time-domain method for the linearized Euler equations," Submitted to the Journal of Computational Physics.

${ }^{10}$ Wang, Y. and Takenaka, H.," A multidomain approach of the Fourier pseudospectral method using discontinuous grid for elastic wave modeling", Earth Planets Space, Vol. 53, 2001, pp. 149-158.

${ }^{11}$ Davies, P.O.A.L and Holland, K.R.,"The Measurement and Prediction of Sound Waves of Arbitrary Amplitude in Practical Flow Ducts," Journal of Sound and Vibration, Vol. 271, 2004, pp. 849-861.

${ }^{12}$ Van der Walt, D.C. ,"Measurement technique to assess the acoustic properties of a silencer component for transient engine conditions," Journal of Sound and Vibration, Vol. 243, 2001, pp. 797-821.

${ }^{13}$ Reymen, Y. ,"3D High-order Discontinuous Galerkin Methods for Time-Domain Simulation of Flow Noise Propagation," Ph.D. thesis, K.U.Leuven, Belgium, 2008.

${ }^{14}$ Silva, F., Guillemain, P., Kergomard, J., Mallaroni, B., Norris A.N., "Approximation formulae for the acoustic radiation impedance of a cylindrical pipe," Journal of Sound and Vibration, Vol. 322, 2009, pp. 255-263.

${ }^{15}$ Levine, H. and Schwinger, J., "On the radiation of sound form an unflanged circular pipe," Physical Review, Vol. 73 , 1948, pp. 383-406. 